\title{
Твердотільна електроніка
}

УДК 537.876 .42

DOI: $10.20535 / 2312-1807.2017 .22 .1 .87570$

Прокопенко Ю. В., д.Т.н., доц. OrcID 0000-0001-6366-9279

e-mail:prok@phbme.kpi.ua

Казмиренко В. А., К.т.н., доц. OrcID 0000-0002-0494-5365

e-mail: v.kazmirenko@phbme.kpi.ua

Чернов А. C., OrcID 0000-0002-5669-9223

e-mail: factit@ukr.net

Национальный технический университет Украины

«Киевский политехнический институт имени Игоря Сикорского»

Ги А. Е. Ванденбош, проф. OrclD 0000-0002-5878-3285

e-mail: guy.vandenbosch@esat.kuleuven.be

Лёвенский Католический Университет

\section{ВЛИЯНИЕ ТОПОЛОГИЧЕСКИХ ПАРАМЕТРОВ НА ДИАПАЗОН ПЕРЕСТРОЙКИ МИКРОМЕХАНИЧЕСКИ УПРАВЛЯЕМЫХ МИКРОПОЛОСКОВЫХ РЕЗОНАТОРОВ}

\begin{abstract}
В работе рассмотрены микромеханически перестраиваемые полосно-заграждающий фиильтр на основе микрополоскового шлейсрового резонатора и полосно-пропускающий фрильтр на основе микрополоскового кольцевого резонатора. Исследовано влияние конструктивно-топологических параметров рассматриваемых фильтров на чувствительность перестройки частоты к микроперемещениям составных частей резонатора. Определена максимально допустимая длина подвижной части шлейфа полосно-заграждающего фильтра на основе шлейфового резонатора, при которой не возникают искажения в рабочей полосе фильтра. Показано влияние ширины подвижной части шлейфра шлейфового резонатора на чувствительность перестройки частоты. Получен критерий выбора ширины подвижной части электрода кольцевого резонатора, а также определено отношение длин подвижной и неподвижной частей, при которых достигается наибольшая возможная перестройки резонансной частоты без искажения частотных характеристик фильтров. Для расчета характеристик структур использовалась методы конечных элементов и эквивалентных параметров.
\end{abstract}

Библ. 12, рис. 10

Ключевые слова: микрополосковый резонатор; кольцевой резонатор; микромеханическая перестройка частоты; полосовой фрильтр.

Введение. Резонаторы СВЧ являются одними из важнейших элементов приемо-передающей аппаратуры. Из-за существования разных стандартов цифровой связи, которые работают в различном диапазоне частот, актуальным является использование перестраиваемых устройств, которые объединяют отдельные селективные устройства. Это позволяет значительно уменьшить массогабаритные характеристики оборудования и затраты на производство. Устройства на основе микро-полосковых линий нашли широкое применение в электронике из-за низкой стоимости производства и миниатюрных размеров. Наиболее распространеными методами перестройки резонансной частоты микрополоскових резонаторов являются использование варкторов
[1], p-i-n-диодов [2], микроелектромеханических систем (MEMC) [3,4]. MEMC, в отличии от варакторов и p-i-n-диодов, обеспечивают больший диапазон перестройки частоты и плавное изменение во всем диапазоне.

В работе [5] предложен принцип перестройки резонансной частоты микрополосковых резонаторов путем изменения эффективной диэлектрической проницаемости линии за счет перемещения сигнального электрода. Требуемые перемещения составляют десятки-сотни микрометров, что позволяет использовать МЭМС и пьезоактюаторы для электромеханического перемещения [6]. В работе [7] показано, что микромеханический способ перестройки обеспечивает перестройку частоты без ухудшения добротности 
и без искажения коэффрициента передачи. Анализ кольцевого резонатора различными методами и способ его перестройки проводились ранее в работах [8-11].

Основные принципы пере-стройки частоты микрополосковых резонаторов микромеханическим методом уже известны и были исследованы ранее [6], но критерии выбора топологических параметров для достижения максимальной чувствительности перестройки ранее не исследовались.

Целью данной работы является исследование влияния топологических параметров микрополосковых резонаторов на их характеристики и определение условий достижения максимальной чувствительности и диапазона перестройки частоты без искажения частотных характеристик.
Влияние длины и ширины подвижной части шлейфа на эффективность частотной перестройки микрополоскового резонатора. Структура микрополоскового шлейфового резонатора представлена на рис. 1. Перестройка частоты осуществляется за счет перемещения подвижной части шлейфра над подложкой. Образовывающийся в результате этого воздушный зазор представляет собой контролируемую неоднородность. Вследствие изменения высоты воздушного зазора $d$, изменяется эффективная диэлектрическая проницаемость управляемого отрезка микрополосковой линии длиной $I_{m}$, что приводит к перестройке резонансной частоты структуры. Часть шлейфра длиной $I_{n}$ остается неподвижной для обеспечения неизменности уровня режекции фрильтра при перестройке.

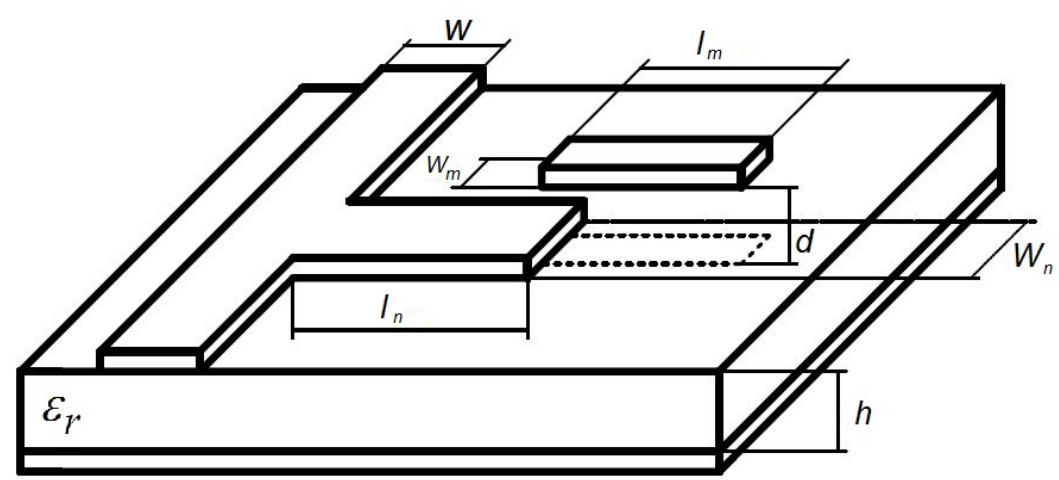

Рис. 1. Структура перестраиваемого шлейфового резонатора на основе микопролосковой линии

Для расчета характеристик фильтра использовались методы конечных элементов (МКЭ) [12] и эквивалентных параметров [6]. Параметры фильтра были выбраны следующими: общая длинна шлейфа $I=20$ мм, ширина электрода $w=1.12 \mathrm{mм}$, толщина электрода $t=5 \mathrm{мкм}$, высота диэлектрической подложки $h=1 \mathrm{~mm}$, диэлектрическая проницаемость подложки $\varepsilon_{r}=10$. На рис. 2 представлена зависимость резонансной частоты от высоты воздушного зазора для разных соотношений подвижной части шлейфа к неподвижной части.

С увеличением воздушного зазора резонансная частота увеличивается и постепенно достигает максимально возможного значения. Это объясняется тем, что при больших воздушных зазорах связь между подвижной и неподвижной частью шлейфа исчезает, и резонансная частота будет определяться только длиной неподвижной части шлейфа. 


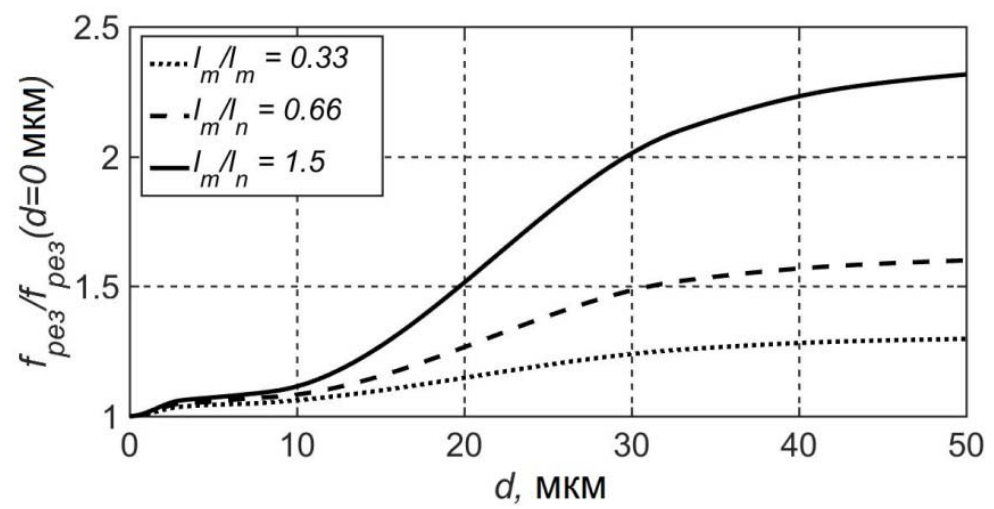

Рис. 2. Зависимость резонансной частоты от высоты воздушного зазора для разных соотношений подвижной и неподвижной частей шлейфа

Как видно из рис. 2, увеличение длины подвижной части шлейфа приводит к повышению эфффективности перестройки частоты. Однако, увеличение длины подвижной части шлейфра имеет ограничения поскольку ее приближение к длине неподвижной части приводит к искаже- нию АЧХ филььтра в связи с появлением дополнительных паразитных резонансных пиков (рис. 3). Это объясняется тем, что при возбуждении структуры, подвижная часть шлейфа также резонирует на частотах, отличных от частот, определенных общей структурой.

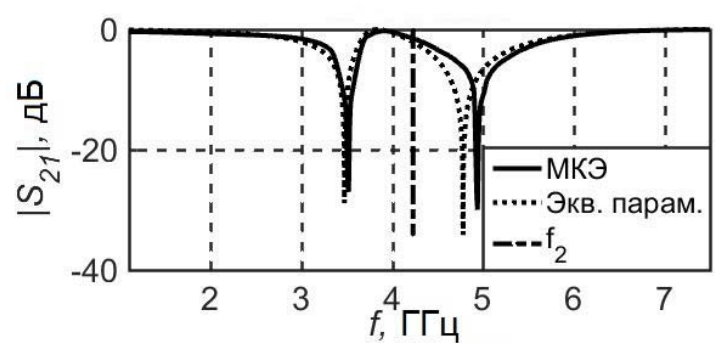

a

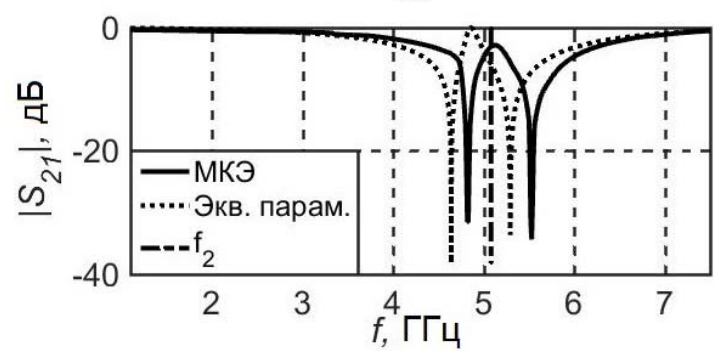

B

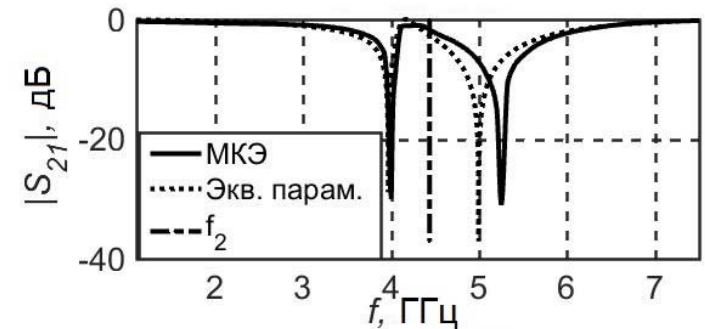

6

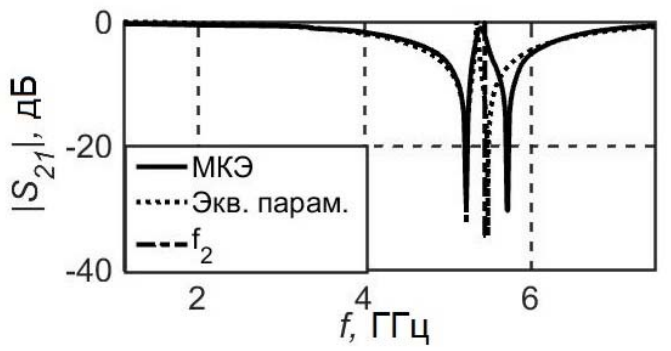

Г

Рис. 3. Зависимость модуля коэффициента передачи от частоты при разных значениях воздушного зазора: а) $d=20$ мкм; б) $d=30$ мкм;

в) $d=70$ мкм; г) $d=100$ мкм;

Паразитные пики появляются вблизи частоты $f_{2}$, равной удвоенной частоте резонанса подвижной части шлейфа $f_{2}=2 f_{m}$. Поскольку максимальная частота перестройки определяется частотой резонанса неподвижной части шлейфа, то для предотвращения появления паразитных пиков необходимо, чтобы выполнялось условие:

$$
f_{n}>f_{m_{-} \min },
$$

где $f_{n}$ - частота резонанса, определяемая неподвижной частью шлейфа; $f_{m \_ \text {min }}$ - минимальная частота резонанса подвижной части шлейфа. 
Частота резонанса шлейфового резонатора определяется по следующей формуле [12]:

$$
f_{\text {рез }}=\frac{c}{\sqrt{\varepsilon_{e \phi p}}} \times \frac{2 n+1}{4 \times I}
$$

где $c$ - скорость света, $I$ - длина шлейфра, $\varepsilon_{э ф р}$ эфрфективная диэлектрическая проницаемость, $n=0,1,2 \ldots$.

Подставляя (2) в (1), получим:

$$
\frac{I_{m}}{I_{n}}<\sqrt{\frac{\varepsilon_{\text {edp_n }} n}{\varepsilon_{\text {ed__m_max }}}},
$$

где $\varepsilon_{\text {эр } n}$-эфрфективная диэлектрическая проницаемость неподвижной части шлейсра, $\varepsilon_{\text {эф_m_max }}-$ максимальная эффрективная диэлектрическая проницаемость подвижной части шлейфра.

Учитывая, что $I=I_{m}+I_{n}$, выражение можно записать следующим образом:

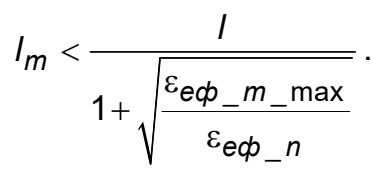

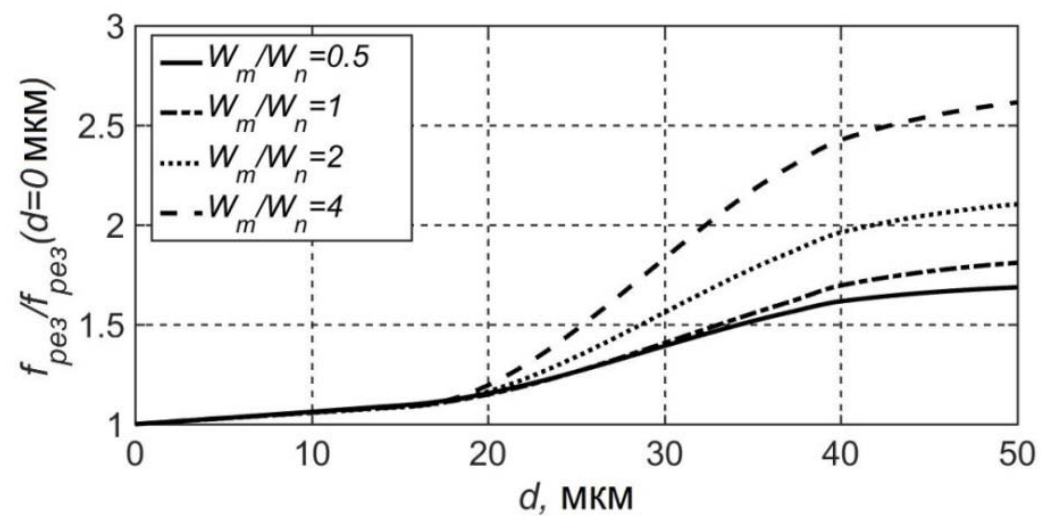

Рис. 4. Зависимость нормированной резонансной частоты

от высоты воздушного зазора для разных значений ширины подвижной части шлейфа

Поскольку, максимальная эфффективная проницаемость подвижной части шлейфа соответствует минимальному воздушному зазору, то чем меньше зазор, тем ближе эфрфективная диэлектрическая проницаемость подвижной части шлейфа к эффрективной диэлектрической проницаемости неподвижной части шлейфра. Таким образом, знаменатель в (4) будет близок к 2, а, значит, максимальная длина подвижной части шлейфа составляет половину общей длины шлейфа.

Длина неподвижной части шлейфра определяется максимальным значением рабочей частоты, которая ограничена условиями возбуждения высших мод. Частота первой высшей моды приблизительно определяется по формуле [12]:

$$
f_{c} \cong \frac{300}{\sqrt{\varepsilon_{r}}(2 w+0.8 h)} .
$$

Принимая во внимание, что максимальная рабочая частота определяется длинной неподвижной части шлейфра и подставляя (1) в (5) получим минимальную длину фриксированной части шлейфра:

$$
I_{n} \geq \frac{c}{\sqrt{\varepsilon_{\Im \varnothing \phi}}} \cdot \frac{\sqrt{\varepsilon_{r}}(2 w+0.8 h)}{400} .
$$

Как видно из (6), минимальная длинна неподвижной части шлейфра зависит от ширины электрода и толщины подложки. Увеличивая ширину электрода или толщину подложки можно уменьшить минимальную длину неподвижной части, тем самым получить более высокие рабочие частоты.

На рис. 4 показана зависимость резонансной частоты от высоты зазора при разных значениях ширины подвижной части шлейфра. Увеличение ширины подвижной части шлейора приводит к увеличению общей чувствительность перестройки, что обусловлено большим изменением эфрфективной диэлектрической проницаемости всей структуры. Поскольку максимальное значение резонансной частоты определяется неподвижной частью шлейфра, которая остается неизменной, то увеличение частотного диапазона перестройки происходит путем уменьшения мини- 
мального значения резонансной частоты, на которую и влияют размеры подвижной части шлейфа.

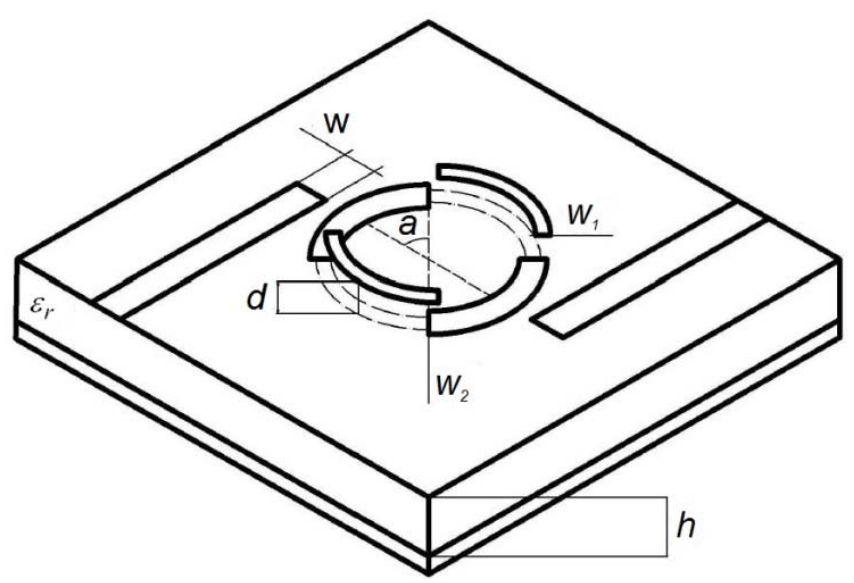

Рис. 5. Структура микромеханически перестраиваемого кольцевого резонатора

Влияние ширины и длин неподвижной и подвижной частей электродов на частотные характеристики кольцевого резонатора. Структура микромеханически перестраиваемого кольцевого резонатора представлена на рис. 5. В отличие от шлейфрового резонатора, кольцевой резонатор имеет две идентичные подвижные ча- сти. Для моделирования использовались следующие топологические параметры: радиус кольца $r=5$ мм, ширина сигнального электрода $w=1.55$ мм, толщина электрода $t=10$ мкм, угол $a=45^{\circ}$, диэлектрическая проницаемость подложки $\varepsilon_{r}=20$, толщина подложки $h=2$ мм.

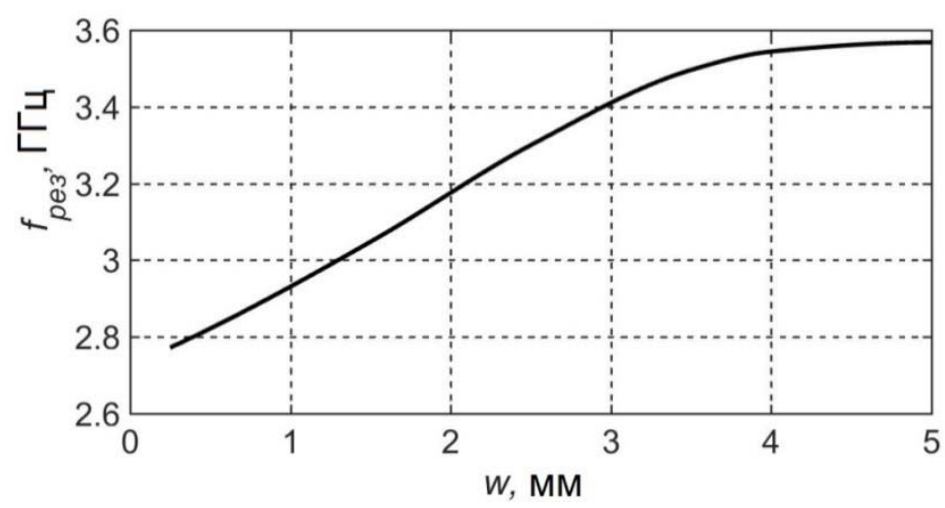

Рис. 6. Зависимость резонансной частоты кольцевого резонатора от ширины кольцевого электрода

На рис. 6 показано влияние ширины кольцевого электрода резонатора на его резонансную частоту. В однородном случае ширина подвижной части кольцевого электрода $\left(w_{1}\right)$ равна ширине неподвижной части кольцевого электрода $\left(w_{2}\right)$.
Ширина кольцевого электрода влияет на эффективность перестройки частоты. В случае очень тонкого кольца перестройка частоты ухудшается. В работе [6] показано, что оптимальное отношение ширины кольцевого электрода к толщине подложки составляет 0.5. 


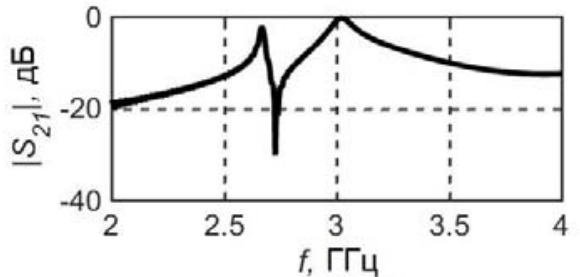

a

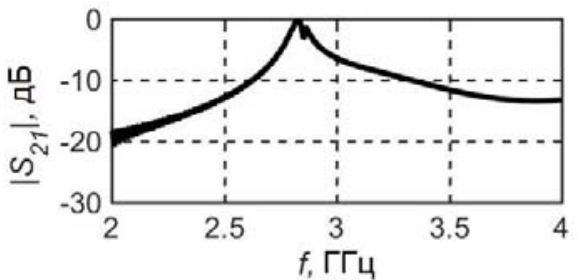

B

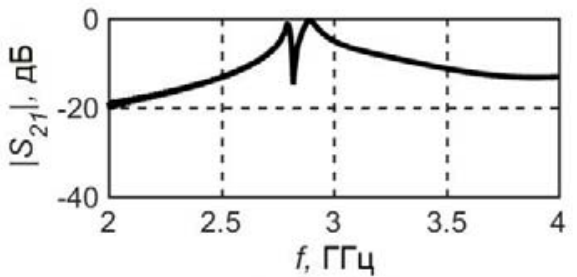

б

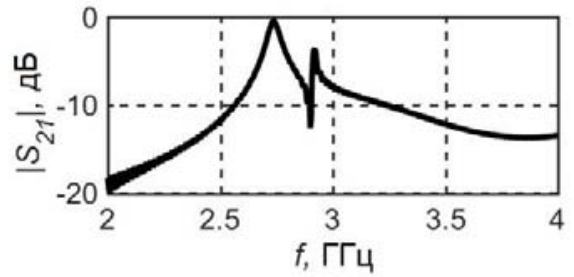

$\Gamma$

Рис. 7. Зависимость коэффрициента передачи кольцевого резонатора от частоты при разных значениях отношения ширины подвижной части кольцевого к неподвижной части:

а) $w_{1} / w_{2}=0.45$; б) $w_{1} / w_{2}=0.81$; в) $w_{1} / w_{2}=1$; г) $w_{1} / w_{2}=1.36$

Однако, в неоднородном случае, когда ширина подвижной части кольцевого электрода $\left(w_{1}\right)$ отличается от ширины неподвижной части кольцевого электрода $\left(w_{2}\right)$, возможно возникновение дополнительного паразитного пика на частотной характеристике. Этот пик возникает изза разности характеристического сопротивления в месте соединения подвижной и неподвижной частей кольцевого электрода. На рис. 7 показано появление дополнительного пика для разных отношений $w_{1}$ и $w_{2}$. С увеличением ширины подвижной части кольцевого электрода, дополнительный пик смещается в область высоких частоты.

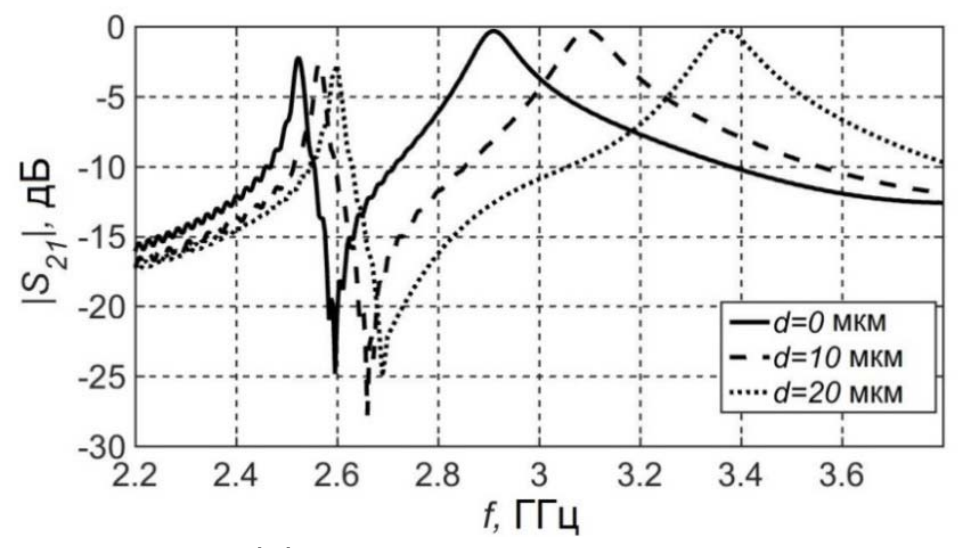

Рис. 8. Зависимость коэффициента передачи кольцевого резонатора от частоты для разных значений воздушного зазора при $w_{1} / w_{2}=0.5$ 


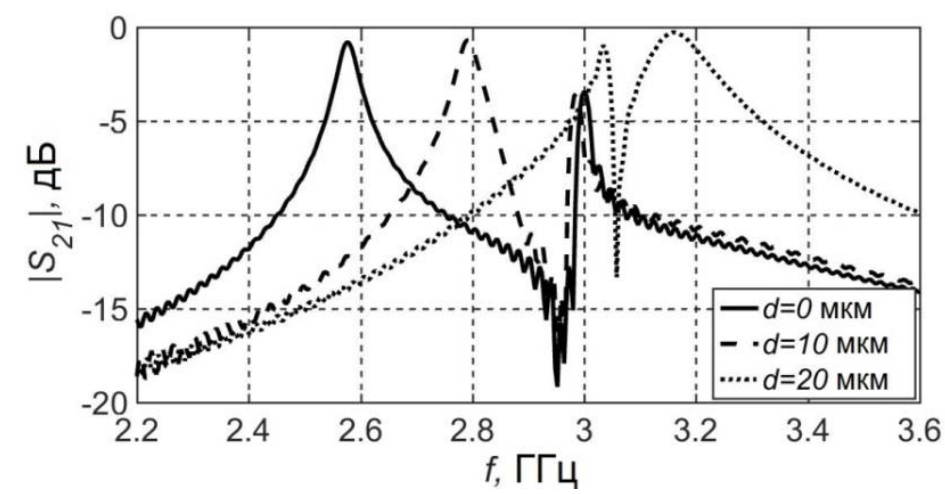

Рис. 9. Зависимость коэффициента передачи кольцевого резонатора от частоты для разных значений воздушного зазора при $w_{1} / w_{2}=1.3$

Стоит отметить, что во время перестройки резонансной частоты, дополнительный пик практически остается на одной частоте для разных значений высоты воздушного зазора. На рис. 8, 9 показаны частотные характеристики для разных отношений ширины подвижной части кольцевого электрода к ширине неподвижной части. Уменьшение этого отношения приводит к смещению паразитного пика в область низких частот, что позволяет исключить его влияние в рабочем диапазоне. Поэтому, желательно выбирать ширину подвижной части кольцевого электрода в два раза меньше, чем ширину неподвижной части.

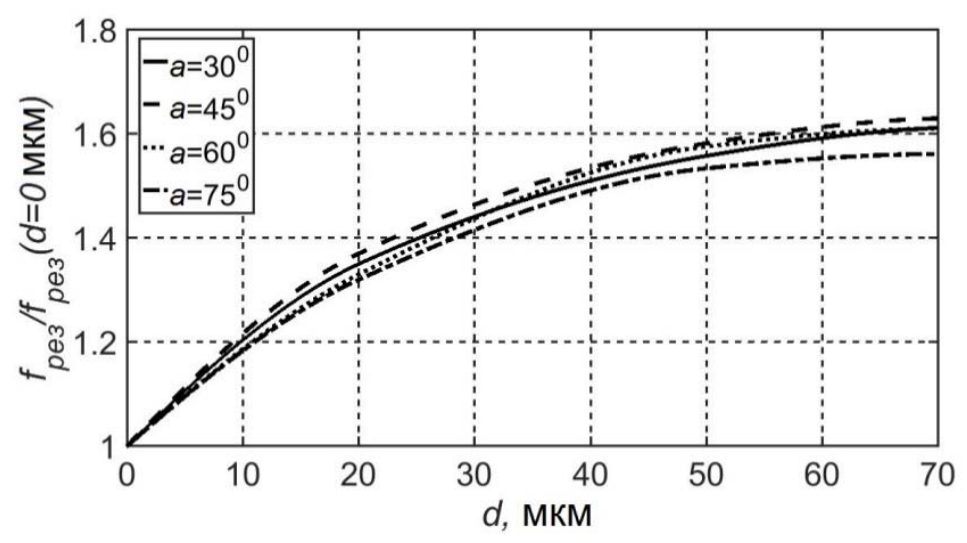

Рис. 10. Зависимость резонансной частоты кольцевого резонатора от высоты воздушного зазора для разных значений соотношений длинны подвижной части к неподвижной части кольцевого электрода

Длины подвижной и неподвижной части кольцевого электрода определяются как длина части круга и зависят от угла а. Увеличение угла а приводит к уменьшению длины подвижной части и увеличению длины неподвижной части и наоборот. На рис. 10 представлена зависимость нормированной резонансной частоты от высоты воздушного зазора. Максимальная чувствительность наблюдается при значении угла а, равном $45^{\circ}$. В этом случае длины подвижной и неподвижной части кольцевого электрода будут равны между собой. Изменение угла $а$, а значит и длины частей, приводит к ухудшению чувствительности перестройки частоты. Представленные зависимости были рассчитаны для случая, когда ширина подвижной части кольцевого электрода равна половине ширины неподвижной части.

Выводы. Увеличение длины подвижной части шлейфрового резонатора, позволяет повысить чувствительность перестройки резонансной частоты. Для достижения максимальной перестройки частоты рекомендуется делать подвижную часть шлейфа равной половине общей длины шлейфра. Превышение данного ограниче- 
ния, приводит к появлению паразитного резонансного пика на частотных характеристиках резонатора. При этом максимальный диапазон перестройки частоты можно увеличить в 2 раза. Уменьшение ширины шлейфового электрода или увеличение высоты подложки, позволяет уменьшить длину неподвижной части шлейфа, а соответственно увеличить общий диапазон перестройки. Увеличение ширины подвижной части шлейфа, также позволяет повысить общую чувствительность перестройки частоты. Повышение диапазона перестройки частоты таким об- разом, достигает практически 70\%, однако, ограничено геометрическими размерамы всей структуры.

Для предотвращения возникновения паразитного пика в рабочем диапазоне частот кольцевого резонатора необходимо делать ширину подвижной части кольцевого резонатора меньше, чем ширина неподвижной части. Существует оптимальное значение соотношения длины подвижной и неподвижной части кольцевого электрода. Для достижения максимальной перестройки частоты длина подвижной части кольцевого электрода должна быть равной длине неподвижной части электрода.

Надійшла до редакції 23 грудня 2016 р.

УДК 537.876 .42

Прокопенко Ю. В., д.Т.н., доц. OrcID 0000-0001-6366-9279

e-mail: prok@phbme.kpi.ua

Казміренко В. А., к.т.Н., доц. OrcID 0000-0002-0494-5365

e-mail: v.kazmirenko@phbme.kpi.ua

Чернов А. C., OrcID 0000-0002-5669-9223

e-mail: factit@ukr.net

Національний технічний університет України

«Київський політехнічний інститут імені Ігоря Сікорського»

Гі А. Е. Ванденбош, проф. OrcID 0000-0002-5878-3285

e-mail: guy.vandenbosch@esat.kuleuven.be

Левенський Католицький Університет

\title{
ВПЛИВ ТОПОЛОГІЧНИХ ПАРАМЕТРІВ НА ДІАПАЗОН ПЕРЕЛАШТУВАННЯ МІКРОМЕХАНІЧНО КЕРОВАНИХ МІКРОСМУЖКОВИХ РЕЗОНАТОРІВ
}

\begin{abstract}
В роботі розглянуті перелаштовувані смуго-загороджуючий фрільтр на основі мікросмужкового шлейфоввого резонатора і смуго-пропускаючий фрільтр на основі мікросмужкового кільцевого резонатора. Досліджено вплив конструктивно-топологічних параметрів розглянутих фрільтрів на чутливість перелаштування частоти до мікропереміщень складових частин резонаторів. Визначена максимальна допустима довжина рухливої частини шлейфу смуго-загороджуючого фрільтру на основі шлейфоового резонатора, за якої не виникають спотворення в робочому діапазоні частот. Показано вплив ширини рухомої частини шлейфру шлейфоового резонатора на чутливість перелаштування частоти. Отримано критерій вибору ширини рухомої частини електрода кільцевого резонатора, а також визначено відношення довжин рухомої та нерухомої частин, за яких досягається найбільше можливе перелаштування резонансної частоти без спотворення частотних характеристик. Для розрахунку характеристик структури використовувались методи скінчених елементів і еквівалентних параметрів.
\end{abstract}

Бібл. 12, рис. 10

Ключові слова: мікросмужковий резонатор; кільцевий резонатор; мікромеханічне перелаштування частоти; смуговий фрільтр. 
UDC 537.876.42

Yu. V. Prokopenko, Dr.Sc. (Eng.), OrcID 0000-0001-6366-9279

e-mail:prok@phbme.kpi.ua

V. A. Kazmirenko, PhD, OrcID OrcID 0000-0002-0494-5365

e-mail: v.kazmirenko@phbme.kpi.ua

A. S. Chernov, OrcID 0000-0002-5669-9223

e-mail: factit@ukr.net

National technical university of Ukraine "Igor Sikorsky Kyiv polytechnic institute"

A. E. Vandenbosch Guy, Full Prof. OrcID 0000-0002-5878-3285

e-mail: guy.vandenbosch@esat.kuleuven.be

Katholieke Universiteit Leuven

\section{INFLUENCE OF TOPOLOGICAL PARAMETERS ON TUNING BAND OF MICROMECHANICALLY CONTROLLED MICROSTRIP RESONATORS}

Tunable band-stop microstrip stub resonator's and band-pass microstrip ring resonator's filters are considered. Influence of structural and topological parameters of the filters on frequency tuning sensitivity to micromovements of resonator components is investigated. Maximal length of stub moving part of band-stop filter based on microstrip stub resonator is defined to achieve frequency tuning without frequency characteristic distortion in operating frequency range. Influence of stub moving part width on frequency tuning sensitivity is demonstrated. A criteria to choose the movable part width of the ring resonator electrode is obtained. Relationship between length of movable and fixed parts to ensure maximal frequency tuning without frequency characteristic distortion is defined. Finite element method (FEM) and equivalent parameters method were used for the structure's frequency characteristics calculation.

Ref. 12, Fig. 10

Keywords: microstrip resonator; ring resonator; micromechanical frequency tuning; band filter

\section{References}

[1]. A.C. Guyette, "Intrinsically Switched Varactor-Tuned Filters and Filter Banks," IEEE Transactions on Microwave Theory and Techniques, vol. 60, no. 4, pp. 1044-1056, 2011,

DOI: 10.1109/TMTT.2012.2184131.

[2]. Z. Brito-Brito, I. Llamas-Garro, G. Navarro-Muñoz, J. Perruisseau-Carrier, L. Pradell et.al., "Precise frequency and bandwidth control of switchable microstrip bandpass filters using diode and microelectro-mechanical system technologies," IET Microwaves, Antennas \& Propagation, vol. 6, pp. 713719, 2012, DOI: 10.1049/iet-map.2012.0077.

[3]. B. Pillans, A. Malczewski, R. Allison and J. Brank, "6-15 GHz RF MEMS Tunable Filters," in IEEE MTT-S International Microwave Digest Symposium, 2005, DOI: 10.1109/MWSYM.2005.1516773.

[4]. B. Pillans, A. Malczewski, F. J. Morris and R. A. Newstrom, "A Family of MEMS Tunable Filters for Advanced RF Applications," in IEEE MTT-S International Microwave Digest Symposium, 2011, DOI: 10.1109/MWSYM.2011.5972889.

[5]. B. B. Pratsiuk, Yu. V. Prokopenko, K. G. Savin and P. Y. Sergiyenko, "Tunable microwave filters based on microstrip lines," Electronics and Communications, no. 4, pp. 53-56, 2011.

[6]. Yu. M. Poplavko, V. M. Pashkov, V. I. Molchanov et. al., "Tunable microwave devices with electromechanical control," Tekhnika i Pribory SVCh, no. 1, pp. 49-59, 2009.

[7]. P. Y. Serhienko, Yu. V. Prokopenko and Yu. M. Poplavko, "Microwave microstrip resonators tuning without quality factor deterioration," Visn. NTUU KPI, Ser. Radioteh. Radioaparatobuduv, no. 56, pp. 74-87, 2014.

[8]. K. Chang, T. S. Martin, F. Wang and J. L. Klein, "On the study of microstrip ring and varactor-tuned ring circuits," IEEE Trans. Microwave Theory and Tech, vol. MTT-35, no. 12, pp. 1288-1295, Dec., 1987, DOI: $10.1109 /$ TMTT.1987.1133850. 
[9]. Wollf and N. Knoppik, "Microstrip ring RSOMtOrS and dispersion measurements on microstrip lines," Electron. Letter, vol. 7, no. 26, pp. 779-781, Dec., 1971, DOI: 10.1049/el:19710532.

[10]. E. Semouchkina, Cao Wenwu and R. Mittra, "FDTD Study of Resonance Processes in microstrip Ring Resonators with Different Excitation Geometries," in Microwave Symposium Digest, 2001 IEEE MTT-S International, 20-25 May, 2001, pp. 2055-2058,

DOI: 10.1109/MWSYM.2001.967316.

[11]. P. Y. Serhienko and Yu. V. Prokopenko, "Ring resonator with micromechanical frequency tuning," Electronics and Communications, no. 4, pp. 23-27, 2012.

[12]. K.C. Gupta and Garg Ramesh, "Microstrip lines and slotlines," Norwood, MA, USA: Artech House, Inc., 1996, 547 p. 